\title{
Practice Spotlight: Pharmacist in a Family Medicine Teaching Unit
}

\author{
Jamie Falk, BScPharm, PharmD \\ Clinical Pharmacist - Primary Care/Family \\ Medicine \\ Kildonan Medical Centre \\ Winnipeg, Manitoba
}

$\mathrm{I}$

n 2008, the pharmacy department of the Winnipeg Regional Health Authority (WRHA) embarked on an initiative to incorporate clinical pharmacy services into a University of Manitoba Department of Family Medicine resident teaching unit. The impetus for the project stemmed from knowledge of national and international experience about the potential benefits of clinical pharmacists in primary care and family medicine environments, a local needs assessment that identified a gap in preventive nonhospital care, and the increasing desire to promote and foster interprofessional collaborative practice. The primary funding source for this initiative was the WRHA, with additional support from the Department of Family Medicine. The clinical pharmacist position (1 full-time equivalent [FTE], divided into 0.8 FTE at the primary site and 0.2 at the secondary sites) has been held by Jamie Falk since July 2008.

The primary site chosen for this position was Kildonan Medical Centre, a family medicine clinic serving a broad range of patients from its location in the northwest corner of Winnipeg, Manitoba. The centre is a resident-run clinic employing 8 attending physicians, 3 nurses, a nurse practitioner, and a clinical pharmacist. The total number of first- and second-year residents in the clinic at any given time ranges from 7 to 12 . A social worker, a dietitian, a psychologist, and a psychiatrist provide services on a part-time basis.

The clinical pharmacy practice model at Kildonan Medical Centre encompasses both direct patient care and teaching roles. Patients are referred to the clinical pharmacist primarily by the attending physicians, residents, and nurses. Medication management for chronic diseases makes up the majority of referrals. Although specific foci identified as areas of need at the start of the project constitute a substantial proportion of the referrals (i.e., cardiovascular risk reduction, type 2 diabetes mellitus, and chronic pain), the clinical pharmacist deals with the wide variety of conditions that might be expected in a busy family medicine clinic. Recommendations resulting from patient assessments are discussed with the patient's attending physician and, when possible and if applicable, the resident and nurse. Evaluation of the first year of this initiative showed that $96 \%$ of the pharmacist's nearly 500 recommendations had been accepted. Discussions with other team members such as the social worker and psychologist have also proven valuable in the management of patients who are being seen concurrently by more than one practitioner, making effective use of the full scope of the multidisciplinary team.

Because of the chronic nature of most of the medical conditions seen by the clinical pharmacist, follow-up visits are needed for the majority of patients. This model naturally poses a challenge in this practice environment. Aside from providing patient care, the clinic's main function is to teach physician residents. As a specialist in pharmacotherapy, the clinical pharmacist has the responsibility not only to provide pharmaceutical care to patients, but also to guide residents. Many of the residents will eventually be working in private practice, without a pharmacist by their side, so this guidance will help them to gain skills in effectively prescribing and monitoring medication therapy.

In the current family medicine teaching model, residents rotate through Kildonan Medical Centre in 6-month cycles. As a result, patients are exposed to new faces on a regular basis. Having a fully dedicated clinical pharmacist in the clinic improves the overall continuity of care. As a primary overseer of medication management, the clinical pharmacist gains a deep understanding of each patient, especially those with more complex medical issues. As a result, this individual is viewed as one of the consistent care providers on whom patients come to rely and who can provide supplemental insight to the team. The dedicated pharmacist position is advantageous not only in terms of availability to patients, but also in terms of availability to staff. This consistent presence has reinforced the concept of clinical pharmacy as an integral part of the interprofessional team.

Direct patient care regularly progresses to informal teaching opportunities during the frequent "hallway consults" requested by residents and other team members. These consults, often extending beyond basic drug information into 
more complex pharmacotherapeutic decision-making, are seen as highly valuable by the multidisciplinary staff members. The resident shares assessment and diagnostic information as the basis for discussions with the clinical pharmacist about appropriate therapy for the patient. These exchanges serve as an important tool for interprofessional learning and the moulding of prescribing practices, with the objective being the advancement of patient care.

In terms of formal teaching, Dr Falk is involved in the more didactic academic half-days for residents, but he also facilitates monthly 1-hour case-based pharmacotherapy sessions with the first-year medical residents and various other learners rotating through the clinic. These sessions represent a valuable opportunity to interact with residents in a nonclinic setting to discuss the nuances of therapy, as well as to help identify specific learning needs.

In a small anonymous survey of Kildonan Medical Centre residents, nearly all (10 of 11) found that the presence of a clinical pharmacist had improved their basic drug knowledge and their knowledge of the literature on chronic disease management, had changed their prescribing practices, had enhanced their overall abilities as a practitioner, and had improved patient care.

Originally inspired by an evidence-based education bulletin produced by Derek Jorgensen in Saskatoon, a bimonthly peer-reviewed pharmacotherapy update is now being produced by Dr Falk, to keep residents and faculty informed about recent developments in the medical literature. The articles in this bulletin are often prompted by newly published studies that have been newsworthy or that have been made so by media coverage. As a result of a desire for broader readership among physicians, the update is now distributed to all of the Department of Family Medicine teaching units for use as a teaching aid with residents. In addition, monthly journal clubs and bimonthly guideline reviews have provided particularly effective opportunities for the clinical pharmacist to gain a reputation as the clinic's evidence-based pharmacotherapy expert. As such, the clinical pharmacist is not simply a provider of drug information, but also plays a key role in clinical decision-making and practice change.
At the time of writing, a diabetes clinic, to be run by the pharmacist and a nurse colleague, was being planned for spring 2011. The focus will be on group education by means of "conversation maps" facilitated by the nurse and pharmacist, followed by separate appointments for each patient with the resident, the dietitian, and a physical activity consultant. The clinic will end with a debriefing session (without patients) to address questions and identify key learning points among the members of the clinic team. In the future, attention will also be given to how the clinical pharmacist might help in ensuring more seamless and synergistic delivery of patient care by Kildonan Medical Centre, its associated community hospital, and the surrounding community pharmacies.

The clinical pharmacy model of practice adopted at Kildonan Medical Centre and the success of other similar models across Canada have prompted further discussion about the expansion of primary care clinical pharmacy services in the WRHA. Currently, strategic planning is under way for the involvement of clinical pharmacy in future community primary care centres in the region. The desire is to have clinical pharmacy move beyond a bonus add-on service to the point where it functions as an essential component of family medicine and primary care teams in the Winnipeg region.

The Practice Spotlight series highlights the accomplishments of Canadian pharmacists with unique practices in hospitals and related health care settings. If you have a unique or innovative practice, or you know someone else who should be profiled, please submit your contact information to Mary Ensom, Editor of CJHP (cjhpedit@cshp.ca), and one of our Associate Editors will be in touch with you. 